\title{
Supplement to "On the Levi-flats in Complex Tori of Dimension Two"
}

By

\author{
Takeo OHSAWA*
}

The purpose of this note is to describe certain Levi-flats in [O] more in detail, by showing that they are natural generalization of Nemirovski's example in $[\mathrm{N}]$.

Let $C$ be a compact Riemann surface, let $L \rightarrow C$ be a holomorphic line bundle, and let $s$ be a meromorphic section of $L$ whose zeros and poles are all simple. Let $C^{\prime} \subset C$ be the complement of the set of zeros and poles of $s$, let $L^{\prime} \subset L$ be the complement of the zero section, and let $\mathbb{R}^{\prime}=\mathbb{R} \backslash\{0\}$.

Let $a>1$, and let $\mathbf{Z}$ act on $L^{\prime}$ by fiberwise multiplication by $a^{m}$ for $m \in \mathbf{Z}$. Then it is easy to see that the closure of $\mathbb{R}^{\prime} \mathrm{s}\left(C^{\prime}\right) / \mathbf{Z}$ in $L^{\prime} / \mathbf{Z}$ is a Levi-flat whose complement is Stein if $C^{\prime} \neq C$ (cf. [N]).

This construction is immediately generalized to produce Levi-flats with Stein complements in higher dimension, by considering smooth ample divisors and the associated line bundles with canonical sections.

On the other hand, since not all elliptic principal bundles arise as quotients of $\mathbb{C}^{*}$-bundles, it may not be so immediate to see how one can generalize Nemirovski's construction to the elliptic principal bundles.

However, it is actually immediate if one regards $d \log s$ as a meromorphic connection of the bundle $L^{\prime} / \mathbf{Z}$ as we shall see below.

Let $C$ be as above, let $E_{0}$ be an elliptic curve, i.e. a compact 1-dimensional complex Lie group, and let $E \stackrel{\pi}{\rightarrow} C$ be a principal $E_{0}$-bundle. Let $\mathfrak{g}$ be the Lie algebra of $E_{0}$. We shall regard $E_{0}$ as a quotient space of $\mathfrak{g}$ by the exponential map. The kernel of the exponential map will be denoted by $\mathfrak{g}_{\mathbf{z}}$ and $E_{0}$ will be identified with $\mathfrak{g} / \mathfrak{g}_{\mathbf{Z}}$ in what follows.

Given a closed subgroup $\Gamma \subset E_{0}$ and a finite subset $\Sigma \subset C$, let $\Omega(\Gamma, \Sigma)$ be the set of meromorphic connections $\omega$ of $E$ with at most simple poles in $\Sigma$

Communicated by K. Saito. Received October 11, 2005.

2000 Mathematics Subject Classification(s): Primary: 32V40; Secondary: 53C40.

* Graduate School of Mathematics, Nagoya University, Chikusaku Furocho, Nagoya, 4648602, Japan.

e-mail: disawa@math.nagoya-u.ac.jp

(C) 2006 Research Institute for Mathematical Sciences, Kyoto University. All rights reserved. 
such that the holonomy group of $\omega$, as that of a holomorphic connection over $C \backslash \Sigma$, preserves $\Gamma$.

For any $\omega \in \Omega\left(E_{0}, \Sigma\right)$ and for any $C^{1}$-curve $\gamma:[0,1] \rightarrow C \backslash \Sigma$, let $\omega_{\gamma}$ denote the parallel transport from $\pi^{-1}(\gamma(0))$ to $\pi^{-1}(\gamma(1))$ with respect to $\omega$ along $\gamma$.

Since $\omega \mid C \backslash \Sigma$ is holomorphic, $\omega_{\gamma}$ depends only on the homotopy class of $\gamma$. Moreover, if $\gamma$ is a closed curve, $\omega_{\gamma}$ is a parallel translate in $\pi^{-1}(\gamma(0))$ defined by addition of an element say $P(\omega, \gamma) \in E_{0} . P(\omega, \gamma)$ is uniquely determined by $\omega$ and the homology class $[\gamma]$ of $\gamma$ in $H_{1}(C \backslash \Sigma, \mathbf{Z})$.

Clearly

$$
\Omega(\Gamma, \Sigma)=\left\{\omega \in \Omega\left(E_{0}, \Sigma\right) \mid P(\omega, \gamma) \in \Gamma \quad \text { if } \quad[\gamma] \in H_{1}(C \backslash \Sigma, \mathbf{Z})\right\} .
$$

It is also clear that $\Omega(\Gamma, \Sigma) \neq \phi$ if the following are satisfied.

(1) $\Omega\left(E_{0}, \Sigma\right) \neq \phi$.

(2) There exist $\omega \in \Omega\left(E_{0}, \Sigma\right)$ and a $\mathfrak{g}$-valued meromorphic 1-form $\sigma$ on $C$ whose poles are all simple and contained in $\Sigma$, such that $\int_{\gamma} \sigma \in P(\omega, \gamma)+\Gamma$ holds for any $[\gamma] \in H_{1}(C \backslash \Sigma, \mathbf{Z})$.

Note that $\Omega\left(E_{0},\left\{P_{0}\right\}\right) \neq \phi$ for any $P_{0} \in C$. To see this, let $U$ be a coordinate neighbourhood of $P_{0}$, and let

$$
\varphi: \pi^{-1}(U) \longrightarrow U \times E_{0}
$$

and

$$
\psi: \pi^{-1}\left(C \backslash\left\{P_{0}\right\}\right) \longrightarrow\left(C \backslash\left\{P_{0}\right\}\right) \times E_{0}
$$

be respectively local trivializations of $E$ over $U$ and $C \backslash\left\{P_{0}\right\}$.

We put $\psi \circ \varphi^{-1}(x, z)=(x, z+c(x))$.

Since $H^{1,1}\left(C,\left[P_{0}\right]\right)=0$, there exist a $\mathfrak{g}$-valued holomorphic 1-form $\tau_{0}$ on $C \backslash\left\{P_{0}\right\}$ and a $\mathfrak{g}$-valued meromorphic 1 -form $\tau_{1}$ on $U$ with at most one simple pole at $P_{0}$ such that

$$
\mathrm{d} c=\tau_{1}-\tau_{0} \quad \text { on } \quad U \backslash\left\{P_{0}\right\} .
$$

Hence $\left\{\tau_{0}, \tau_{1}\right\}$ gives a meromorphic connection of $E \rightarrow C$ with possibly one simple pole at $P_{0}$. It is clear that the period of the primitive of $\tau_{1}$ around $P_{0}$ does not depend on the choice of $\varphi$ and $\psi$. We shall call this element of $\mathfrak{g}$ the curvature of the bundle $E$. Clearly the curvature of $E$ is zero if $E$ is flat.

Therefore, $\Omega(\Gamma, \Sigma) \neq \phi$ if the curvature preserves $\Gamma$ and $\operatorname{deg} \Sigma$ is sufficiently large, since one may choose then a g-valued meromorphic 1-form $\tau$ on $C$ with simple poles in $\Sigma \cup\left\{P_{0}\right\}$ in such a way that the holonomy of the connection $\left\{\tau_{0}+\tau, \tau_{1}+\tau\right\}$ preserves $\Gamma$. 
Moreover, if the pair $\left(\left\{\tau_{0}, \tau_{1}\right\}, \Gamma\right)$ satisfies this condition, there exists $n_{0} \in$ $\mathbf{N}$ such that, for any $n \geq n_{0}$ one can find $\Sigma$ with $\Omega(\Gamma, \Sigma) \neq \phi$ and $\operatorname{deg} \Sigma=n$ that additionally satisfies the following properties:

1) The curve $\oint_{P_{0}} t\left(\tau_{1}+\tau\right)(0 \leq t \leq 1)$ is orthogonal to $\Gamma$ at $t=0$ with respect to the conformal metric and intersects with $\Gamma$ precisely at the endpoints $(t=0$ and $t=1)$.

2) $\oint_{Q}\left(\tau_{0}+\tau\right)= \pm \oint_{P_{0}}\left(\tau_{1}+\tau\right)$ for any $Q \in \Sigma$.

Here $\oint_{\#} *$ denotes the period of the primitive of $*$ around \#.

In particular, if $\oint_{P_{0}} \tau_{1}$ preserves $\Gamma$, one can find $\Sigma$ and a meromorphic connection $\omega=\left\{\tau_{0}+\tau, \tau_{1}+\tau\right\}$ in such a way that, for any $P \in C \backslash \Sigma \backslash\left\{P_{0}\right\}$ and for any $\tilde{P} \in \pi^{-1}(P)$, the closure of the set

$$
\bigcup_{\gamma} \omega_{\gamma}(\tilde{P}+\Gamma)
$$

is a real analytic Levi flat hypersurface in $E$ which contains $\pi^{-1}\left(\Sigma \cup\left\{P_{0}\right\}\right)$. Here $\gamma$ runs through $C^{1}$-curves on $C \backslash \Sigma \backslash\left\{P_{0}\right\}$ starting from $P$.

We shall denote this hypersurface by $X(E, \Gamma, \omega, P)$ and call it a Levi-flat of Nemirovski type.

To see that Nemirovski's example is a special case of Levi-flats of Nemirovski type, one may put $E=L^{\prime} / \mathbf{Z}$ with $E_{0}=\mathbb{C}^{*} / \mathbf{Z}, \Gamma=\mathbb{R}^{\prime} / \mathbf{Z}, \omega=d \log s$ and $\tilde{P}=s(P)(\neq 0, \infty)$.

The following is immediate from the definition of Levi-flats of Nemirovski type.

Theorem 1. Let $E \rightarrow C$ be an $E_{0}$-principal bundle with curvature $\xi \in$ g. Then

(I) If $\xi=0, X(E, \Gamma, \omega, \tilde{P})$ exists for any $\Gamma$.

(II) If $\xi \neq 0, X(E, \Gamma, \omega, P)$ exists if and only if $\sqrt{-1} \mathbb{R} \xi \cap \mathfrak{g}_{\mathbf{Z}} \neq\{0\}$.

$X(E, \Gamma, \omega, P)$ will be called of type $I$ (resp. of type $I I)$ if $\xi=0$ (resp. if $\xi \neq 0)$. Then the classification result in $[\mathrm{O}]$ for the Levi-flats in 2 -tori can be stated in the following way.

Theorem 2. $\quad$ Let $T$ be a complex 2-torus with a flat metric and let $X \subset$ $T$ be a connected real analytic Levi-flat. Then one of the following holds.

(i) X is flat. 
(ii) There exists an elliptic curve $C$ and a surjective holomorphic map $T \rightarrow C$ with connected fibers such that either $X$ is the preimage of a simple closed real curve in $C$, or $X$ is a Levi-flat of Nemirovski type of type I.

\section{References}

[N] Nemirovski, S., Stein domains with Levi-flat boundaries on compact complex surfaces, Math. Notes, 66 (1999), 522-525.

[O] Ohsawa, T., On the Levi-flats in complex tori of dimension two, Publ. RIMS, Kyoto Univ., 42 (2006), 361-377. 